\title{
Asymptotic Controllability and Input-to-State Stabilization: The Effect of Actuator Errors*
}

\author{
Michael Malisoff ${ }^{1}$ and Eduardo Sontag ${ }^{2}$ \\ 1 Department of Mathematics, Louisiana State University and A. \& M. College, \\ Baton Rouge LA 70803-4918. malisoff@lsu.edu \\ 2 Department of Mathematics, Rutgers University, New Brunswick NJ 08901. \\ sontag@control.rutgers.edu
}

Summary. We discuss several issues related to the stabilizability of nonlinear systems. For a given continuously stabilizable system, we review some constructions of feedbacks that render the system input-to-state stable with respect to actuator errors. We also announce a new feedback design that makes globally asymptotically controllable systems input-to-state stable to actuator errors and small observation noise. We illustrate our constructions using the nonholonomic integrator, and we discuss a related feedback design for systems with disturbances.

\section{Introduction}

The theory of input-to-state stable (ISS) systems forms the basis for much modern nonlinear feedback design and analysis (cf. $[9,10,11,12,14,26])$. In this survey, we deal with several issues related to the input-to-state stabilizability of nonlinear finite-dimensional continuous-time control systems. We focus on some relatively new feedback designs for asymptotically controllable systems that render the corresponding closed-loop systems ISS with respect to actuator errors. We cover the following topics:

1. Basic controllability and stability concepts

2. Continuously stabilizable systems with actuator errors

3. Asymptotic controllability implies input-to-state stabilization

4. Feedback stabilization of the nonholonomic integrator

5. Systems with large observation noise

6. Integral-input-to-state stabilization

One of our main tools will be the existence theory for smooth controlLyapunov functions (CLF's) for continuously stabilizable systems. We also use a recent extension of this theory that provides semiconcave CLF's for

\footnotetext{
* Sponsors: Louisiana Board of Regents Contract LEQSF(2002-04)-ENH-TR-26 (Malisoff); USAF Grant F49620-01-1-0063, NSF Grant CCR-0206789 (Sontag).
} 
systems which are merely asymptotically controllable. Our emphasis will be on relatively new areas of research, including the integral-input-to-state stabilization of systems with disturbances (cf. $[1,13])$. While our discussion will be mainly conceptual, we will refer the reader to the relevant literature, where detailed statements and proofs of all our results are found. For a survey on the underlying theory of CLF's, stability, and stabilization, see [25]. As a general reference, we refer the reader to the second author's preprints at http://www . math.rutgers. edu/ sontag/papers.html.

\section{Basic Controllability and Stability Concepts}

In much of what follows, we deal with a general nonlinear finite-dimensional continuous-time deterministic system of the form

$$
\dot{x}=f(x, u)
$$

evolving over Euclidean space, where $x(t) \in \mathbb{R}^{n}$ for all $t \geq 0$ and the controls $u$ (which are also called inputs) are measurable essentially bounded functions $u:[0, \infty) \rightarrow \mathcal{U}=\mathbb{R}^{m}$ taking their values in the control set $\mathcal{U}$ which we have taken to be $\mathbb{R}^{m}$. We denote the set of all inputs for (1) by $\mathcal{M}^{m}$. When we wish to restrict the size of controls $u \in \mathcal{M}^{m}$ in the essential supremum $|\cdot|_{\infty}$, we will also use the sets $\mathcal{M}_{N}^{m}=\left\{u \in \mathcal{M}^{m}:|u|_{\infty} \leq N\right\}$, defined for each $N>0$. Many of our results easily generalize to locally essentially bounded inputs mapping into more general control sets $\mathcal{U} \subseteq \mathbb{R}^{m}$. When we refer to (1), we always assume that $f$ is locally Lipschitz and that $f(0,0)=0$. We also study systems with no inputs

$$
\dot{x}=f(x)
$$

for continuous $f$; all definitions for such systems are implicitly applied to (1) by setting $u=0$, e.g., we define global asymptotic stability (GAS) for (2), but we say (1) is GAS if the corresponding zero-input system $\dot{x}=f(x, 0)$ is GAS.

We denote the solution of (1) starting at any initial state $x_{o}$ for any given control $u \in \mathcal{M}^{m}$, defined on its maximal interval, by $x\left(\cdot, x_{o}, u\right)$. We let $|\cdot|$ denote the Euclidean norm, and we set $r \mathcal{B}_{k}:=\left\{x \in \mathbb{R}^{k}:|x|<r\right\}$ for each $k \in \mathbb{N}$ and $r>0$. We denote the closure of $r \mathcal{B}_{k}$ by $r \overline{\mathcal{B}}_{k}$. When we say that a function is smooth, we mean that it is $C^{1}$ (i.e., continuously differentiable). We call a function $\alpha: \mathbb{R}^{k} \rightarrow[0, \infty)$ proper (a.k.a. radially unbounded) if $\alpha(x) \rightarrow+\infty$ as $|x| \rightarrow+\infty$. We say that a function $\alpha: \mathbb{R}^{k} \rightarrow[0, \infty)$ is positive definite provided $\alpha(x)=0$ iff $x=0$.

We will frequently use the following types of comparison functions. We let $\mathcal{K}_{\infty}$ denote the set of all continuous functions $\rho:[0, \infty) \rightarrow[0, \infty)$ for which (i) $\rho(0)=0$ and (ii) $\rho$ is strictly increasing and unbounded. Also, we let $\mathcal{K} \mathcal{L}$ denote the set of all continuous functions $\beta:[0, \infty) \times[0, \infty) \rightarrow[0, \infty)$ for which (1) $\beta(\cdot, t) \in \mathcal{K}_{\infty}$ for each $t \geq 0$, (2) $\beta(s, \cdot)$ is nonincreasing for each 
$s \geq 0$, and (3) $\beta(s, t) \rightarrow 0$ as $t \rightarrow+\infty$ for each $s \geq 0$. We also use $\mathcal{N}$ to denote the set of all nondecreasing functions $\sigma:[0, \infty) \rightarrow[0, \infty)$.

In terms of comparison functions, the property of global asymptotic stability (GAS) of the system (2) without inputs is as follows:

$$
(\exists \beta \in \mathcal{K} \mathcal{L}) \quad|x(t)| \leq \beta\left(\left|x_{o}\right|, t\right)
$$

for all $x_{o} \in \mathbb{R}^{n}$, all trajectories $x(\cdot)$ of the system (2) starting at $x_{o}$, and all $t \geq 0$. More generally, we say that the system (1) with inputs is globally asymptotically controllable (GAC) provided:

$$
\begin{aligned}
& (\exists \beta \in \mathcal{K} \mathcal{L})(\exists \sigma \in \mathcal{N}) \quad \forall x_{o} \in \mathbb{R}^{n}, \exists u(\cdot) \\
& |u|_{\infty} \leq \sigma\left(\left|x_{o}\right|\right),\left|x\left(t, x_{o}, u\right)\right| \leq \beta\left(\left|x_{o}\right|, t\right) \quad \forall t \geq 0 .
\end{aligned}
$$

Roughly speaking, our definition of GAC amounts to requiring that for each initial state, there exists a control such that the corresponding solution is defined on $[0, \infty)$ and converges to zero with 'small overshoot' and also that the input remains bounded for $x$ near zero.

The notion of input-to-state stable (ISS) systems provides an alternative generalization of GAS, in which all trajectories of (1) converge to zero with an overshoot depending on the sup norm of the input. The ISS property was introduced in [20], and has become a fundamental concept on which much modern nonlinear feedback analysis and design are based (cf. [9, 10, 11, 12, $14]$ ). By definition, a dynamics $\dot{x}=f(x, u)$ is ISS (with respect to $u$ ) provided

$$
(\exists \beta \in \mathcal{K} \mathcal{L})\left(\exists \gamma \in \mathcal{K}_{\infty}\right)|x(t)| \leq \beta\left(\left|x_{o}\right|, t\right)+\gamma\left(|u|_{\infty}\right)
$$

for all $x_{o} \in \mathbb{R}^{n}$, all $u \in \mathcal{M}^{m}$, all trajectories $x(\cdot)$ of the dynamics for $u$ starting at $x_{o}$, and all $t \geq 0$. In much of what follows, we will be studying systems

$$
\dot{x}=f(x, K(x)+u)
$$

where $K$ is a specified feedback map for (1) and the input $u \in \mathcal{M}^{m}$ represents an actuator error. By a (memoryless state) feedback, we mean a locally bounded function $K: \mathbb{R}^{n} \rightarrow \mathcal{U}$ satisfying $K(0)=0$. For the special case of the dynamics (4) and continuous $K$, the ISS property (3) implies that $K$ is a stabilizing feedback (meaning that the closed-loop system $\dot{x}=f(x, K(x)$ ) is GAS) and also that the perturbed system (4) exhibits bounded behavior for arbitrary (essentially) bounded actuator errors $u \in \mathcal{M}^{m}$ (but see also $\S 4$ for the definition of trajectories for ISS systems with discontinuous controllers). We say that (1) is input-to-state stabilizable (a.k.a. ISSable) provided there exists a feedback $K$ such that (4) is ISS. We say that (1) is continuously stabilizable (a.k.a. $C^{o}$-stabilizable) provided there exists a continuous feedback $K$ such that $\dot{x}=f(x, K(x))$ is GAS. Clearly, $C^{o}$-stabilizable systems are GAC, but not conversely (cf. $\S \S 4-5$ ).

In general, saying that a continuous dynamics $\dot{x}=f(x, u)$ is ISS implies that the corresponding zero-input system $\dot{x}=f(x, 0)$ is GAS. This suggests the following natural question: 
Question 1. If (1) is $C^{o}$-stabilizable, is it also ISSable?

We will address this question in $§ 3$. More generally, it is natural to ask:

Question 2. If (1) is GAC, is it also ISSable?

We will address Question 2 in $\S 4$. We will see that the answers to these questions are yes, provided the given system (1) is control-affine, i.e., if it has the form $\dot{x}=h(x)+G(x) u$. For the general fully nonlinear system (1), we will see that the answers to these questions are still yes if the system is transformed using a feedback equivalence (cf. $\S 3$ for the relevant definitions).

However, if the system is merely GAC, then there may be obstructions to continuous (time-invariant) feedback stabilization (cf. [3, 14, 25]). Therefore, we will need to allow discontinuous feedbacks. This produces the technical problem of defining precisely what is meant by a solution of the dynamics when $\dot{x}=f(x, K(x)+u)$ is not continuous, since the standard Carathéodory existence theorems for solutions would not apply. We will resolve this problem by interpreting the trajectories of (4) for discontinuous $K$ as Euler solutions. The preceding issues are central to the rest of this survey.

\section{$3 C^{o}$-Stabilizable Systems with Actuator Errors}

In this section, we review the main results from $[20,22]$ on the input-tostate stabilizability of the system (1). Throughout this section, we assume the system (1) is continuously stabilizable (i.e., $C^{o}$-stabilizable), meaning, there exists a continuous feedback $K_{1}$ for which

$$
\dot{x}=f\left(x, K_{1}(x)\right)
$$

is GAS. We wish to design a continuous feedback function $K$ (which we allow to be different from the feedback used in (5)) rendering the perturbed system $\dot{x}=f(x, K(x)+u)$ ISS with respect to the actuator error $u$. This design problem plays an important role in control applications, where one wishes to have a feedback that is robust to noise in the input channel.

For a fully nonlinear system, such a feedback fails to exist in general. For example, consider the scalar system $\dot{x}=-x+x^{2} u^{2}$. By considering closedloop trajectories starting at $x_{o}=4$ for the input $u \equiv 1$ (cf. [22]), one can check that there is no continuous feedback $K$ for which the closed-loop system

$$
\dot{x}=-x+x^{2}(K(x)+u)^{2}
$$

is ISS, even though $\dot{x}=-x$ is obviously GAS (i.e., using $K_{1}(x) \equiv 0$ as the stabilizer in (5)). In fact, if we interpret the trajectories of (6) in the more general sense of sampling and Euler solutions (cf. $\S 4$ for the definitions), then one can show that there is no discontinuous feedback rendering (6) ISS either. 
On the other hand, if we assume the given system (1) has the control-affine form

$$
\dot{x}=h(x)+G(x) u,
$$

then we have the following positive result shown in [20]:

Theorem 1. If (7) is $C^{o}$-stabilizable, then there exists a continuous feedback $K: \mathbb{R}^{n} \rightarrow \mathbb{R}^{m}$ for which

$$
\dot{x}=h(x)+G(x)(K(x)+u)
$$

is ISS.

Theorem 1 follows by choosing $K(x)=K_{1}(x)-\nabla V(x) G(x)$, where $V$ is a $C^{1}$ control-Lyapunov function (CLF) for (7) and $K_{1}$ is a continuous feedback rendering (7) GAS. The existence of such a CLF was established in [2]. (See also $[17,18]$ for the existence of semiconcave CLF's for GAC systems, and [25] or $\S 4$ below for the definition of CLF's, in terms of set-valued differentials.)

While the example (6) illustrates that $C^{o}$-stabilizability does not in general imply input-to-state stabilizability, we can still extend Theorem 1 to fully nonlinear systems by using the following weaker notion of ISS that was introduced in [22]: We say that the system (1) is ISSable in the weak sense (a.k.a. weak ISSable) provided there exist a continuous feedback $K$, and an $m \times m$ matrix $\Theta$ of continuously differentiable functions which is invertible at each point, such that $\dot{x}=f(x, K(x)+\Theta(x) u)$ is ISS. We then have the following extension of Theorem 1 for fully nonlinear systems shown in [22]:

Theorem 2. If (1) is $C^{o}$-stabilizable, then it is ISSable in the weak sense.

Theorem 2 can be restated in terms of feedback equivalence, as follows. Recall that two systems $\dot{x}=f(x, u)$ and $\dot{x}=\hat{f}(x, u)$ evolving on $\mathbb{R}^{n} \times \mathbb{R}^{m}$ are said to be feedback equivalent provided that there exist a feedback $K: \mathbb{R}^{n} \rightarrow \mathbb{R}^{m}$ and an everywhere invertible function $\Theta: \mathbb{R}^{n} \rightarrow \mathbb{R}^{m \times m}$ for which $\hat{f}(x, u)=f(x, K(x)+\Theta(x) u)$ for all $x \in \mathbb{R}^{n}$ and $u \in \mathbb{R}^{m}$; the systems are said to be continuously feedback equivalent if, in addition, $K$ can be taken to be continuous. (See also [24] for a more general definition of feedback equivalence, also involving a diffeomorphic transformation of the state variable.) The following is then a direct consequence of Theorem 2:

Corollary 1. The fully nonlinear control system (1) is $C^{o}$-stabilizable if and only if it is continuously feedback equivalent to an ISS system.

See also $[5,7]$ for a construction of time-varying ISS stabilizing feedback.

\section{Asymptotic Controllability Implies ISS Stabilization}

In the last section, we saw how $C^{o}$-stabilizable systems can be stabilized with respect to actuator errors. However, in many applications, the given system is 
not $C^{o}$-stabilizable. For example, if $m<n$ and $\operatorname{rank}\left[g_{1}(0), g_{2}(0), \ldots, g_{m}(0)\right]=$ $m$, then it is impossible to continuously stabilize the drift-free system $\dot{x}=$ $u_{1} g_{1}(x)+\ldots+u_{m} g_{m}(x), x \in \mathbb{R}^{n}$; thus, no totally nonholonomic mechanical system is $C^{o}$-stabilizable (cf. [25], p.560). On the other hand, drift-free systems are typically GAC (cf. $\S 5$ for an example).

This motivates our study of the more general situation where the given control-affine system $\dot{x}=h(x)+G(x) u$ is GAC, but not necessarily $C^{o}$ stabilizable. We wish to design a feedback $K$ so that the closed-loop system

$$
\dot{x}=h(x)+G(x)(K(x)+u)
$$

is ISS. In fact, we will design our feedback for (9) so that the more general closed-loop system

$$
\dot{x}=h(x)+G(x)(K(x+e)+u)
$$

is ISS with respect to the actuator error $u$ when the observation error $e$ is sufficiently small (cf. definitions below). In this context, the precise values of $e(t)$ are unknown to the controller, but information about upper bounds on $|e(t)|$ can be used to design the feedback. For the case of continuous feedbacks $K$, small errors in the controller in (10) can be tolerated. However, if we allow discontinuous $K$, then small observation errors can have a substantial effect on the dynamics, in which case the magnitude of $e$ will need to be constrained in terms of the frequency of the sampling (cf. below for the precise restrictions on e). For a construction of a stabilizing feedback $K$ for (10) under the stronger assumption that the given system is $C^{o}$-stabilizable, see [25].

Since GAC systems are not in general $C^{o}$-stabilizable, we will in fact need to consider discontinuous feedbacks for (10), which produces the technical problem of defining precisely what is meant by a solution for a system that is discontinuous in the state, since the usual existence theorems for solutions of differential equations would not apply. Our solutions will therefore be taken in the more general sense of sampling and Euler solutions. By an Euler solution, we mean a uniform limit of sampling solutions, taken as the frequency of sampling becomes infinite. The following definitions from [14] make these ideas precise.

We say that $\pi=\left\{t_{o}, t_{1}, t_{2}, \ldots\right\} \subset[0, \infty)$ is a partition provided $t_{o}=0$, $t_{i}<t_{i+1}$ for all $i \geq 0$, and $t_{i} \rightarrow+\infty$ as $i \rightarrow+\infty$. The set of all partitions is denoted by Par. Let $F: \mathbb{R}^{n} \times \mathbb{R}^{m} \times \mathbb{R}^{m} \rightarrow \mathbb{R}^{n}:(x, p, u) \mapsto F(x, p, u)$ be a continuous function which is locally Lipschitz in $x$ uniformly on compact subsets of $\mathbb{R}^{n} \times \mathbb{R}^{m} \times \mathbb{R}^{m}$. Recall that a feedback (for $F$ ) is defined to be any locally bounded function $K: \mathbb{R}^{n} \rightarrow \mathbb{R}^{m}$ for which $K(0)=0$. In particular, we allow discontinuous feedbacks. The arguments $x, p$, and $u$ in $F$ represent the state, feedback value, and actuator error, respectively.

We set $\mathcal{O}:=\left\{e:[0, \infty) \rightarrow \mathbb{R}^{n}\right\}$ and $\sup (e):=\sup \{|e(t)|: t \geq 0\}$ for all $e \in \mathcal{O}$, and $\mathcal{O}_{\eta}:=\{e \in \mathcal{O}: \sup (e) \leq \eta\}$ for each $\eta>0$. Given a feedback $K: \mathbb{R}^{n} \rightarrow \mathbb{R}^{m}$, an initial value $x_{o} \in \mathbb{R}^{n}$, a partition $\pi=\left\{t_{o}, t_{1}, t_{2}, \ldots\right\} \in$ Par, $e \in \mathcal{O}$, and $u \in \mathcal{M}^{m}$, the sampling solution for the initial value problem 


$$
\begin{aligned}
\dot{x}(t) & =F(x(t), K(x(t)+e(t)), u(t)) \\
x(0) & =x_{o}
\end{aligned}
$$

is the continuous function defined by recursively solving

$$
\dot{x}(t)=F\left(x(t), K\left(x\left(t_{i}\right)+e\left(t_{i}\right)\right), u(t)\right)
$$

from the initial time $t_{i}$ up until time

$$
s_{i}=t_{i} \vee \sup \left\{s \in\left[t_{i}, t_{i+1}\right]: x(\cdot) \text { is defined on }\left[t_{i}, s\right)\right\},
$$

where $x(0)=x_{o}$ (cf. $\left.[4,14]\right)$. The sampling solution of (11)-(12) is defined from time zero up to the maximal time $\bar{t}=\inf \left\{s_{i}: s_{i}<t_{i+1}\right\}$. This sampling solution will be denoted by $t \mapsto x_{\pi}\left(t ; x_{o}, u, e\right)$ to exhibit its dependence on $\pi \in \operatorname{Par}, x_{o} \in \mathbb{R}^{n}, u \in \mathcal{M}^{m}$, and $e \in \mathcal{O}$, or simply by $x_{\pi}$ when the dependence is clear from the context. In particular, if $s_{i}=t_{i+1}$ for all $i$, then $\bar{t}=+\infty$, so in that case, the sampling solution $t \mapsto x_{\pi}\left(t ; x_{o}, u, e\right)$ is defined on $[0, \infty)$.

We also define the upper diameter and lower diameter of a given partition $\pi=\left\{t_{o}, t_{1}, t_{2}, \ldots\right\} \in$ Par by

$$
\overline{\mathbf{d}}(\pi)=\sup _{i \geq 0}\left(t_{i+1}-t_{i}\right), \quad \underline{\mathbf{d}}(\pi)=\inf _{i \geq 0}\left(t_{i+1}-t_{i}\right)
$$

respectively. We let $\operatorname{Par}(\delta):=\{\pi \in \operatorname{Par}: \overline{\mathbf{d}}(\pi)<\delta\}$ for each $\delta>0$. We say that a function $y:[0, \infty) \rightarrow \mathbb{R}^{n}$ is an Euler solution of (11)-(12) (robust to small observation errors) for $u \in \mathcal{M}^{m}$ provided there are sequences $\pi_{r} \in$ Par and $e_{r} \in \mathcal{O}$ such that

(a) $\overline{\mathbf{d}}\left(\pi_{r}\right) \rightarrow 0 ; \quad$ (b) $\sup \left(e_{r}\right) / \underline{\mathbf{d}}\left(\pi_{r}\right) \rightarrow 0$; and

(c) $t \mapsto x_{\pi_{r}}\left(t ; x_{o}, u, e_{r}\right)$ converges uniformly to $y$

as $r \rightarrow+\infty$. In terms of sampling solutions, we have the following analog of ISS from [14]:

Definition 1. We say that (11) is ISS for sampling solutions provided there exist $\beta \in \mathcal{K} \mathcal{L}$ and $\gamma \in \mathcal{K}_{\infty}$ satisfying: For each $\varepsilon, M, N>0$ with $0<\varepsilon<M$, there exist positive $\delta=\delta(\varepsilon, M, N)$ and $\kappa=\kappa(\varepsilon, M, N)$ such that for each $\pi \in \operatorname{Par}(\delta), x_{o} \in M \overline{\mathcal{B}}_{n}, u \in \mathcal{M}_{N}^{m}$, and $e \in \mathcal{O}$ for which $\sup (e) \leq \kappa \underline{\mathbf{d}}(\pi)$,

$$
\left|x_{\pi}\left(t ; x_{o}, u, e\right)\right| \leq \beta(M, t)+\gamma(N)+\varepsilon
$$

for all $t \geq 0$.

Notice that condition (13) is defined to hold in particular for $M=\left|x_{o}\right|$ and $N=|u|_{\infty}$. Conceptually, condition (13) says that the system is ISS, modulo small overflows, if the sampling is done 'quickly enough' to satisfy $\pi \in \operatorname{Par}(\delta)$, but 'not too quickly', as determined by the additional requirement that $\underline{\mathbf{d}}(\pi) \geq(1 / \kappa) \sup (e)$. Moreover, if we restrict to the case where $e=0$, then the condition on $\underline{\mathbf{d}}(\pi)$ in Definition 1 is no longer needed. Notice that the bounds on $e$ are in the supremum, not the essential supremum.

We also use the following analog of Definition 1 for Euler solutions: 
Definition 2. We say that the system (11) is ISS for Euler solutions provided there are $\beta \in \mathcal{K} \mathcal{L}$ and $\gamma \in \mathcal{K}_{\infty}$ satisfying: If $u \in \mathcal{M}^{m}$ and $x_{o} \in \mathbb{R}^{n}$ and $t \mapsto x(t)$ is an Euler solution of (11)-(12), then

$$
|x(t)| \leq \beta\left(\left|x_{o}\right|, t\right)+\gamma\left(|u|_{\infty}\right)
$$

for all $t \geq 0$.

The following result on control-affine systems is shown in [14]:

Theorem 3. If (7) is GAC, then there exists a feedback $K$ for which the corresponding closed-loop system (10) is ISS for sampling and Euler solutions.

The proof of Theorem 3 is based on the existence theory for semiconcave CLF's from [17]-[18], which we review next. Let $\Omega \subseteq \mathbb{R}^{n}$ be open. A continuous function $g: \Omega \rightarrow \mathbb{R}$ is called semiconcave on $\Omega$ provided for any point $x_{o} \in \Omega$, there exist $\rho, C>0$ such that

$$
g(x)+g(y)-2 g\left(\frac{x+y}{2}\right) \leq C\|x-y\|^{2}
$$

for all $x, y \in x_{o}+\rho \mathcal{B}_{n}$. The proximal superdifferential (resp., proximal subdifferential) of a function $V: \Omega \rightarrow \mathbb{R}$ at $x \in \Omega$, which is denoted by $\partial^{P} V(x)$ (resp., $\partial_{P} V(x)$ ), is the set of all $\zeta \in \mathbb{R}^{n}$ for which there exist $\sigma, \eta>0$ such that $V(y)-V(x)-\sigma|y-x|^{2} \leq\langle\zeta, y-x\rangle$ (resp., $\left.V(y)-V(x)-\sigma|y-x|^{2} \geq\langle\zeta, y-x\rangle\right)$ for all $y \in x+\eta \mathcal{B}_{n}$. The limiting subdifferential of a continuous function $V: \Omega \rightarrow \mathbb{R}$ at $x \in \Omega$ is

$$
\partial_{L} V(x):=\left\{\begin{array}{l}
q \in \mathbb{R}^{n}: \text { there exist } x_{n} \rightarrow x \text { and } \\
q_{n} \in \partial_{P} V\left(x_{n}\right) \text { such that } q_{n} \rightarrow q .
\end{array}\right\} .
$$

In terms of proximal subdifferentials, a CLF is defined as follows (cf. [25]):

Definition 3. A control-Lyapunov function (CLF) for (1) is a continuous, positive definite, proper function $V: \mathbb{R}^{n} \rightarrow \mathbb{R}$ for which there exist a continuous, positive definite function $W: \mathbb{R}^{n} \rightarrow \mathbb{R}$ and $\alpha \in \mathcal{N}$ satisfying

$$
\forall \zeta \in \partial_{P} V(x), \quad \inf _{|u| \leq \alpha(|x|)}\langle\zeta, f(x, u)\rangle \leq-W(x)
$$

for all $x \in \mathbb{R}^{n}$.

The existence of continuous CLF's for GAC systems was established in [19]. On the other hand, the papers [17]-[18] establish the following stronger existence result: If (1) is GAC, then there exists a CLF $V$ for (1) that is semiconcave on $\mathbb{R}^{n} \backslash\{0\}$ and $\alpha \in \mathcal{N}$ that satisfy

$$
\forall \zeta \in \partial_{L} V(x), \min _{|u| \leq \alpha(|x|)}\langle\zeta, f(x, u)\rangle \leq-V(x)
$$


for all $x \in \mathbb{R}^{n}$. Theorem 3 follows from this stronger existence result by first taking an arbitrary selection $\zeta(x) \in \partial_{L} V(x)$ for $x \neq 0$, and then using the combined feedback $K=K_{1}+K_{2}$, where for each $x \neq 0, K_{1}(x) \in \alpha(|x|) \mathcal{B}_{m}$ is any vector $u$ satisfying the inequality in (15) for $\zeta=\zeta(x)$, and

$$
K_{2}(x)=-V(x)\left(\begin{array}{c}
\operatorname{sgn}\left(\left\langle\zeta(x), g_{1}(x)\right\rangle\right) \\
\operatorname{sgn}\left(\left\langle\zeta(x), g_{2}(x)\right\rangle\right) \\
\vdots \\
\operatorname{sgn}\left(\left\langle\zeta(x), g_{m}(x)\right\rangle\right)
\end{array}\right)
$$

where $g_{i}$ is the $i$ th column of the matrix $G$ and

$$
\operatorname{sgn}(s)=\left\{\begin{aligned}
1, & s>0 \\
-1, & s<0 \\
0, & s=0
\end{aligned}\right.
$$

It then follows that the closed-loop system (10) is ISS for sampling and Euler solutions, using the combined feedback $K=K_{1}+K_{2}$ (with $K(0)=0$ ).

Here is a sketch of the proof that this combined feedback renders the system ISS for sampling solutions when the observation error $e=0$. (See [14] for the proof of the general case, and for the proof that this feedback also gives ISS for Euler solutions.) In what follows, $\|u(s)\|_{J}$ denotes the essential supremum of the restriction of a function $u$ to an interval $J$. Let $M, N>0$ be given, and $V$ be a semiconcave CLF for the given dynamics, as above. Define the functions $\underline{\alpha}, \bar{\alpha} \in \mathcal{K}_{\infty}$ by

$$
\underline{\alpha}(s):=\min \{s, \min \{|x|: V(x) \geq s\}\} \bar{\alpha}(s):=\max \{|x|: V(x) \leq s\} .
$$

Let $x \mapsto \zeta(x)$ be any selection as above and $\zeta(0) \in \mathbb{R}^{n}$ be arbitrary.

For each $x \in \mathbb{R}^{n}$, we can choose $u=u_{x} \in \alpha(|x|) \mathcal{B}_{m}$ that satisfies the inequality in (15) for the given dynamics. Set $F(x, p, u):=f(x)+G(x)(p+u)$. Choose $S:=\left\{x \in \mathbb{R}^{n}: V(x) \leq \underline{\alpha}^{-1}(N)\right\}$ and $\varepsilon \in(0, \min \{1, M\})$ for which $(2 \varepsilon) \mathcal{B}_{n} \subseteq S$. Set

$$
\begin{aligned}
& Q:=\left\{\left[\bar{\alpha} \circ \underline{\alpha}^{-1}(N+M)+1\right] \overline{\mathcal{B}}_{n}\right\} \backslash \varepsilon \mathcal{B}_{n}, \\
& \lambda_{-}:=\min \left\{V(p): p \in Q^{\varepsilon / 2}\right\}, \quad \lambda_{+}:=\max \left\{V(p): p \in Q^{\varepsilon}\right\} .
\end{aligned}
$$

It follows that $S \subseteq Q^{\varepsilon}$. We can then choose $\tilde{\varepsilon} \in(0, \varepsilon)$ for which

$$
\bar{\alpha}\left(p+\frac{\mathcal{L}_{\varepsilon}}{4} \tilde{\varepsilon}\right) \leq \bar{\alpha}(p)+\frac{\varepsilon}{8} \forall p \in\left[0, \underline{\alpha}^{-1}(N)+\lambda_{+}\right]
$$

where $\mathcal{L}_{\varepsilon}>1$ is a Lipschitz constant for $V$ on $Q^{\varepsilon / 2}$. Using local uniformity properties for semiconcave functions (cf. [14]), we can find $\sigma, \mu>0$ such that

$$
V(y)-V(x) \leq\langle\zeta(x), y-x\rangle+\sigma|y-x|^{2}
$$


for all $y \in x+\mu \mathcal{B}_{n}$ and $x \in Q^{\varepsilon / 2}$. We can then choose

$$
\begin{gathered}
\delta=\delta(\varepsilon, M, N) \in\left(0, \frac{\tilde{\varepsilon}}{16+\lambda_{+}+16 \lambda_{+}}\right) \text {such that } \\
\left|x_{\pi}\left(t ; x_{o}, u, 0\right)-x_{i}\right| \leq \min \left\{\mu, \frac{\tilde{\varepsilon}}{16\left(1+\mathcal{L}_{\varepsilon}\right)}, \sqrt{\frac{\lambda_{-}}{8 \sigma}\left(t-t_{i}\right)}\right\}
\end{gathered}
$$

(where $\left.x_{i}=x_{\pi}\left(t_{i} ; x_{o}, u, 0\right)\right)$ and

$$
\begin{aligned}
& \left\|\zeta\left(x_{i}\right) \cdot\left(F\left(x_{i}, K\left(x_{i}\right), u(s)\right)-f\left(x_{\pi}(s)\right)-G\left(x_{\pi}(s)\right)\left[u(s)+K\left(x_{i}\right)\right]\right)\right\|_{J_{i}} \\
& \leq \frac{\lambda_{-}}{8}
\end{aligned}
$$

where $J_{i}:=\left[t_{i}, t_{i+1}\right]$, for all $u \in \mathcal{M}_{N}^{m}, t \in\left[t_{i}, t_{i+1}\right], \pi \in \operatorname{Par}(\delta)$, and all $i$ such that $x_{i} \in Q^{\varepsilon / 2}$. Defining $J(t):=16 /(16+t)$ and

$$
\beta(s, t):=\bar{\alpha}\left(\underline{\alpha}^{-1}(s) J(t)\right), \gamma(s):=\bar{\alpha} \circ \underline{\alpha}^{-1}(s),
$$

we can then use the estimates (20)-(21) to conclude that the sampling ISS estimate (13) holds for all $x_{o} \in M \overline{\mathcal{B}}_{n}, u \in \mathcal{M}_{N}^{m}, \pi \in \operatorname{Par}(\delta)$, and $e=0$.

The preceding construction uses the control-affine structure of the given GAC system (7) in an essential way. In fact, it is not difficult to construct examples of fully nonlinear GAC systems $\dot{x}=f(x, u)$ for which there is no feedback $K$ rendering the closed-loop system $\dot{x}=f(x, K(x)+u)$ ISS for sampling and Euler solutions. One such example was provided by (6), p.4.

On the other hand, it is possible to extend Theorem 3 to fully nonlinear systems if we interpret ISS in terms of feedback equivalence, as follows. In what follows, we assume for simplicity that the observation error in the controller $e \equiv 0$. Recall (cf. [14]) that if $f$ and $\hat{f}$ are feedback equivalent, and if $K$ and $\Theta$ satisfy the requirements of our definition of feedback equivalence, then we also say that $\dot{x}=f(x, u)$ is feedback equivalent to (11) with the choice $F(x, p, u)=f(x, p+\Theta(x) u)$. We then have the following corollary from [14]:

Corollary 2. The fully nonlinear control system (1) is GAC if and only if it is feedback equivalent to a system which is ISS for sampling and Euler solutions.

For a proof of this corollary, see [14].

\section{Feedback Stabilization of the Nonholonomic Integrator}

In this section, we use the feedback construction from $\S 4$ to ISS stabilize Brockett's nonholonomic integrator control system. The nonholonomic integrator was introduced in [3] as an example of a system that cannot be stabilized using continuous state feedback. It is well-known that if the state space of a system contains topological obstacles (e.g., if the state space is $\mathbb{R}^{2} \backslash(-1,1)^{2}$, 
and therefore has a topological obstacle around the origin), then it is impossible to stabilize the system using continuous state feedback. This follows from a theorem of Milnor, which asserts that the domain of attraction of an asymptotically stable vector field must be diffeomorphic to Euclidean space.

Brockett's example illustrates how, even if we assume that the state evolves in Euclidean space, obstructions to continuous stabilization may still occur. These obstructions are not due to the topology of the state space, but instead arise from the form of the control system. Such obstacles occur when it is impossible to move instantly in some directions, even though it is possible to move eventually in every direction; when this is the case, we call the dynamics nonholonomic (cf. [25]). This gives rise to Brockett's criterion, which was first announced in [3]. Brockett's criterion is a necessary condition for the existence of a continuous (time-invariant) feedback stabilizer for (1); it requires that the mapping $(x, u) \mapsto f(x, u)$ be open at zero. For linear systems $\dot{x}=A x+B u$, this says $\operatorname{rank}[A, B]=n$, which is the Hautus controllability condition at the zero mode (cf. [25]). The nonholonomic integrator does not satisfy Brockett's criterion, and therefore cannot be stabilized by continuous state feedbacks.

The physical model for Brockett's example is as follows. Consider a threewheeled shopping cart whose front wheel acts as a castor. The state variable is $\left(x_{1}, x_{2}, \theta\right)^{T}$, where $\left(x_{1}, x_{2}\right)^{T}$ is the midpoint of the rear axle of the cart, and $\theta$ is the cart's orientation. The front wheel is free to rotate, but there is a "nonslipping" constraint that $\left(\dot{x}_{1}, \dot{x}_{2}\right)^{T}$ must always be parallel to $(\cos (\theta), \sin (\theta))^{T}$. This gives the equations $\dot{x}_{1}=v_{1} \cos (\theta), \dot{x}_{2}=v_{1} \sin (\theta)$, and $\dot{\theta}=v_{2}$, where $v_{1}$ is a 'drive' command and $v_{2}$ is a steering command. Using a feedback transformation (cf. [25], or $\S 4.3$ in [24]) brings the equations into the form

$$
\dot{x}_{1}=u_{1}, \quad \dot{x}_{2}=u_{2}, \quad \dot{x}_{3}=x_{1} u_{2}-x_{2} u_{1},
$$

which is called the nonholonomic integrator control system.

One can show that (23) is a GAC system. However, since Brockett's condition is not satisfied for (23), the system has no continuous state feedback stabilizer. While there does not exist a $C^{1}$ CLF for the system (23), it is now well-known that every GAC system admits a continuous CLF (see [19]). In fact, it was shown in [16] that the dynamics (23) has the continuous CLF

$$
V(x)=\max \left\{\sqrt{x_{1}^{2}+x_{2}^{2}},\left|x_{3}\right|-\sqrt{x_{1}^{2}+x_{2}^{2}}\right\},
$$

which is semiconcave outside the cone $x_{3}^{2}=4\left(x_{1}^{2}+x_{2}^{2}\right)$. On the other hand, it is not difficult to show (cf. [14]) that the system (23) also has the CLF

$$
\hat{V}(x)=\left(\sqrt{x_{1}^{2}+x_{2}^{2}}-\left|x_{3}\right|\right)^{2}+x_{3}^{2},
$$

which is semiconcave on $\mathbb{R}^{3} \backslash\{0\}$. This allows us to apply the theory of the previous section to the nonholonomic integrator with the CLF (25). To check the semiconcavity of $\hat{V}$, it suffices to verify that 


$$
S(x)=-\left|x_{3}\right| \sqrt{x_{1}^{2}+x_{2}^{2}}
$$

is semiconcave on $\mathbb{R}^{3} \backslash\{0\}$, since $C^{2}$ functions are semiconcave and the sum of two semiconcave functions is semiconcave. On the other hand, the semiconcavity of $S$ follows easily because $\phi(r, s)=-|r s|=\min \{ \pm r s\}$ is semiconcave, as a minimum of two $C^{2}$ functions. The proof that $\hat{V}$ is a CLF follows from a slight variant of the change of coordinate arguments used in [16] to show that (24) is a CLF; we leave the proof to the reader as an exercise.

For the nonholonomic integrator dynamics and the CLF (25), the stabilizing $K$ from $\S 4$ is constructed as follows. The system vector fields are

$$
h(x)=0, \quad G(x)=\left(\begin{array}{cc}
1 & 0 \\
0 & 1 \\
-x_{2} & x_{1}
\end{array}\right)
$$

and we can define $\zeta(\cdot)$ by $\zeta(0)=0$ and

$\zeta(x)= \begin{cases}2\left(\left|x_{3}\right|-r(x)\right)\left(-x_{1} / r(x),-x_{2} / r(x), \operatorname{sgn}\left(x_{3}\right)\right)+2 x_{3} \mathbf{e}_{3}, & x_{3} r(x) \neq 0, \\ -2 r(x)\left(-x_{1} / r(x),-x_{2} / r(x), 1\right), & x_{3}=0, \\ 2\left|x_{3}\right|\left(0,-1, \operatorname{sgn}\left(x_{3}\right)\right)+2 x_{3} \mathbf{e}_{3}, & r(x)=0\end{cases}$

for all $x \neq 0$, where $r(x):=\sqrt{x_{1}^{2}+x_{2}^{2}}$ and $\mathbf{e}_{3}=(0,0,1)^{T}$. In terms of

$$
b(x)=\zeta(x) G(x)= \begin{cases}\left(\begin{array}{ll}
\mu(x)\left(-x_{1} / r(x)-x_{2} \operatorname{sgn}\left(x_{3}\right)\right)-2 x_{2} x_{3} \\
\mu(x)\left(-x_{2} / r(x)+x_{1} \operatorname{sgn}\left(x_{3}\right)\right)+2 x_{1} x_{3}
\end{array}\right), & r(x) x_{3} \neq 0 \\
-2 r(x)\left(\begin{array}{l}
-x_{1} / r(x)-x_{2} \\
-x_{2} / r(x)+x_{1}
\end{array}\right), & x_{3}=0 \\
\left(\begin{array}{c}
0 \\
-2\left|x_{3}\right|
\end{array}\right), & r(x)=0\end{cases}
$$

where $\mu(x):=2\left(\left|x_{3}\right|-r(x)\right)$, the stabilizing feedback is then

$$
K(x)=-V(x)\left(\frac{b(x)}{|b(x)|^{2}}+\left(\operatorname{sgn}\left(b_{1}(x)\right), \operatorname{sgn}\left(b_{2}(x)\right)\right)\right)
$$

for $x \neq 0$ and $K(0)=0$. It follows that the corresponding closed-loop system $\dot{x}=h(x)+G(x)(K(x+e)+u)$ is ISS for sampling and Euler solutions, using the feedback $(26)$.

\section{Systems with Large Observation Noise}

In the preceding section, we studied the problem of constructing a state feedback $K$ such that the closed-loop system

$$
\dot{x}=h(x)+G(x)(K(x+e)+u)
$$


is ISS with respect to the actuator error $u$, where $e$ is an observation noise. We assumed that the given system $\dot{x}=h(x)+G(x) u$ was GAC, and we also used the fact that the observation noise $e$ in the controller was sufficiently small. It is natural to ask whether our analysis can be extended to the case where the observation error $e$ is an arbitrary bounded measurable function. More generally, one could consider the following conjecture (where we use $w$ to denote the input to distinguish it from the actuator error):

Conjecture $L$. If $h: \mathbb{R}^{n} \rightarrow \mathbb{R}^{n}$ and $G: \mathbb{R}^{n} \rightarrow \mathbb{R}^{n \times m}$ are locally Lipschitz with $h(0)=0$, and if $\dot{x}=h(x)+G(x) w$ is $C^{o}$-stabilizable, then there exists a continuous feedback $K: \mathbb{R}^{n} \rightarrow \mathbb{R}^{m}$ satisfying the following: There exist $\beta \in \mathcal{K} \mathcal{L}$ and $\gamma_{1}, \gamma_{2} \in \mathcal{K}_{\infty}$ such that for each initial value $x_{o} \in \mathbb{R}^{n}$ and each $e \in \mathcal{M}^{n}$ and each $u \in \mathcal{M}^{m}$, each solution $x(t)$ of the closed-loop system (27) starting at $x_{o}$ satisfies $|x(t)| \leq \beta\left(\left|x_{o}\right|, t\right)+\gamma_{1}\left(|u|_{\infty}\right)+\gamma_{2}\left(|e|_{\infty}\right)$ for all $t \geq 0$.

An important difference between this problem and the previous one is that the "small" observation noise $e$ is now allowed to be large, but we have relaxed the stabilization objective to an ISS one. This problem is of interest in the context of observer design. For details, see [25].

It turns out that Conjecture $\mathrm{L}$ is false, even if the effect of actuator errors $u$ is ignored. A counterexample to the conjecture for a single input plant was provided in [6]. The counterexample is based on the $C^{o}$-stabilizable system

$$
\dot{x}=\left(I+2 \Theta\left(\frac{\pi}{2}\right) x x^{T}\right) \Theta\left(x^{T} x\right) \cdot\left(\left[\begin{array}{cc}
-1 & 0 \\
0 & x^{T} x
\end{array}\right] \Theta\left(-x^{T} x\right) x+\left[\begin{array}{l}
0 \\
1
\end{array}\right] w\right),
$$

where $x \in \mathbb{R}^{2}$ and $\Theta$ is the rotation matrix

$$
\Theta(\theta)=\left[\begin{array}{rr}
\cos \theta & -\sin \theta \\
\sin \theta & \cos \theta
\end{array}\right] .
$$

One can show that there is no continuous feedback $K$ for this system that can satisfy the requirements of Conjecture L.

This can be seen by choosing the actuator error $u \equiv 0$ and expressing the system in terms of the variable $z:=\Theta\left(-x^{T} x\right) x$, and then considering only initial conditions that lie on the invariant set $S=\left\{x \in \mathbb{R}^{2}: z_{1}=0\right\}$. One then constructs a sensor disturbance $e$ with the property that the corresponding value for the perturbed state $x(t)+e(t)$ would lie on $S \cap\left\{z_{2}<0\right\}$ when the actual state $x(t)$ lies on $S \cap\left\{z_{2}>0\right\}$ (cf. [6] for details). See [8] for a construction of continuous feedback rendering $\dot{x}=f(x)+G(x) K(x+e)$ ISS with respect to $e$, which applies to a restricted class of $C^{o}$-stabilizable single input nonlinear systems in lower triangular form. See also [7] for timevarying feedbacks that make control-affine systems ISS to observation errors; the feedbacks in [7] apply for scalar systems, and for systems that satisfy a feedback passivity property. 


\section{Integral-Input-to-State Stabilization}

In the previous sections, we constructed feedbacks that render systems ISS to actuator errors. A system that is ISS exhibits low total energy or low overshoot when excited by energy bounded or uniformly bounded signals, respectively. While these are highly desirable characteristics, it is sometimes the case that feedback design does not render ISS behavior, or that a property weaker than ISS is verified in a step in a recursive design. One such weaker property, which was introduced in [23], is integral-input-to-state stability (iISS). The iISS condition reflects the qualitative property of having small overshoots when the disturbances have finite energy. It provides a nonlinear analog of "finite $H^{2}$ norm" for linear systems, and as such has obvious physical relevance and significance.

In this section, we study the integral-input-to-state stabilizability of

$$
\dot{x}=h(x, d)+G(x) u
$$

where $u \in \mathcal{M}^{m}$ is an input, $d \in \mathcal{M}^{m}$ is a disturbance, and the functions $h$ and $G$ are locally Lipschitz with $h(0,0)=0$. This includes control-affine systems with no disturbances, as a special case. We wish to design a state feedback $k$ for which the closed-loop system

$$
\dot{x}=h(x, d)+G(x) k(x),
$$

exhibits iISS. This design problem is related to the problems in the previous sections, because $k$ will be used to mitigate the effect of the error term $d$. We will require that $k$ be almost smooth, i.e., smooth on $\mathbb{R}^{n} \backslash\{0\}$ and continuous on $\mathbb{R}^{n}$. Recall from [13] that the iISS condition for (30) states that there exist $\alpha_{o}, \gamma \in \mathcal{K}_{\infty}$ and $\beta \in \mathcal{K} \mathcal{L}$ such that for each disturbance $d \in \mathcal{M}^{m}$ and initial value $x_{o} \in \mathbb{R}^{n}$, each solution $x(t)$ of (30) starting at $x_{o}$ satisfies

$$
\alpha_{o}(|x(t)|) \leq \beta\left(\left|x_{o}\right|, t\right)+\int_{0}^{t} \gamma(|d(s)|) \mathrm{d} s
$$

for all $t \geq 0$. As pointed out in [13, 23], iISS is a weaker requirement than ISS; for example, the scalar system $\dot{x}=-x+x d$ is iISS but not ISS.

The feedback constructions in the preceding sections are based on the existence of CLF's. In parallel to those results, the feedback $k$ for (30) can be constructed using an iISS-CLF for (29), which is defined as follows (cf. [13]):

Definition 4. We say that a positive definite radially unbounded smooth function $V: \mathbb{R}^{n} \rightarrow \mathbb{R}$ is an iISS-CLF for the system (29) provided there exist a positive definite function $\alpha: \mathbb{R} \rightarrow[0, \infty)$ and a class $\mathcal{K}_{\infty}$ function $\chi$ such that

$$
\inf _{u \in \mathbb{R}^{m}}\{a(x, d)+b(x) u\} \leq-\alpha(|x|)+\chi(|d|) \quad \forall x \in \mathbb{R}^{n}, d \in \mathbb{R}^{m},
$$

where $a(x, d)=\nabla V(x) h(x, d)$ and $b(x)=\nabla V(x) G(x)$. 
Our construction of the feedback $k$ is based on the well-known 'universal formula' for feedback stabilization, which was introduced in [21] and later generalized to $p$-norm bounded controls in [15]. We will use the functions

$$
K_{u}(a, b):=- \begin{cases}\frac{a+\sqrt{a^{2}+|b|^{4}}}{|b|^{2}} b, & b \neq 0 \\ 0, & b=0\end{cases}
$$

and

$$
\omega(x):=\max _{d}\{a(x, d)-\chi(|d|)\}
$$

where $a(x, d)$ and $\chi$ are from the iISS-CLF definition, and where we assume without loss of generality that $\chi(r) / r \rightarrow+\infty$ as $r \rightarrow+\infty$, which is necessary for $\omega$ to be well-defined. The function $K_{u}$ comes from the 'universal' stabilizing formula from [21] (cf. Remark 1 for details). In terms of the functions $\alpha$ and $b$ from Definition 4, we say that an iISS-CLF $V$ has the small control property (scp) provided: for each $\varepsilon>0$, there exists $\delta>0$ such that if $0<|x|<\delta$, then there exists $u$ with $|u|<\varepsilon$ for which $\omega(x)+b(x) u \leq-\alpha(|x|)$. We also choose an almost smooth function $\bar{\omega}$ that satisfies

$$
\omega(x)+\alpha(|x|) / 3 \leq \bar{\omega}(x) \leq \omega(x)+2 \alpha(|x|) / 3 \quad \forall x \in \mathbb{R}^{n},
$$

where $\alpha$ is the positive definite function from the definition of an iISS-CLF. The existence of $\bar{\omega}$ is standard (cf. [13]). In terms of the functions we have defined, we have the following feedback construction shown in [13]:

Theorem 4. If $V$ is an iISS-CLF for (29) satisfying the small control property, then the feedback $k(x):=K_{u}(\bar{\omega}(x), b(x))$ is almost smooth and renders the closed-loop system (30) integral-input-to-state stable.

The proof of Theorem 4 is based on the iISS-Lyapunov function characterizations from [1].

Remark 1. In addition to the preceding construction, formula (32) can also be used to give an explicit expression for our feedback $K=K_{1}+K_{2}$ from $\S 3$, under the additional assumption that the CLF $V$ for the given system $\dot{x}=h(x)+G(x) u$ satisfies the small control property (cf. [21]). Indeed, in this case, it suffices to use the feedback $K_{1}(x)=K_{u}(a(x), b(x))$ and $K_{2}$ as before, where $a(x)=\nabla V(x) h(x)$ and $b(x)=\nabla V(x) G(x)$ are the Lie derivatives in the direction of $V$. When defined in this way, $K_{1}$ is the so-called universal formula for feedback stabilization from [21], and is almost smooth. A similar construction can be made when the given system is merely GAC (cf. §4); in this case, we replace the Lie derivatives $a(x)$ and $b(x)$ in the formula for $K_{1}$ with $\tilde{a}(x)=\zeta(x) h(x)$ and $\tilde{b}(x)=\zeta(x) G(x)$, respectively, for a selection $\zeta(x) \in \partial_{L} V(x)$ for the (possibly nonsmooth, but semiconcave) CLF.

Remark 2. The novelty of Theorem 4 is that it uses a universal formula, rather than the more commonly used pointwise min norm control laws (which are 
in general just continuous) or partition of unity arguments (which are nonconstructive). For extensions of Theorem 4 to systems with outputs, see [13]. The proof of Theorem 4 is based on the fact that the disturbance $d$ and the input $u$ in (29) are decoupled. This allows the interchange of the order of the max from the definition of $\omega$ and the inf in the decay condition (31) (cf. [13], §5). Consequently, the proof breaks down for systems where $u$ and $d$ are coupled. The search for extensions of the theorem to more general systems is an important question that is of considerable ongoing research interest.

\section{References}

1. Angeli D, Sontag E, Wang Y (2000) A characterization of integral input-to-state stability, IEEE Trans Automat Control 45:1082-1097

2. Artstein Z (1983) Stabilization with relaxed controls, Nonlinear Analysis 11:1163-1173

3. Brockett R (1983) Asymptotic stability and feedback stabilization. In: Brockett R, Millman R, Sussmann H (eds) Differential Geometric Control Theory. Birkhäuser Boston

4. Clarke F, Ledyaev Y, Sontag E, Subbotin A (1997) Asymptotic controllability implies feedback stabilization, IEEE Trans Automat Control 42:1394-1407

5. Fah N (1999) Input-to-state stability with respect to measurement disturbances for one-dimensional systems, ESAIM Control Optim Calc Var 4:99-121

6. Freeman R (1995) Global internal stabilizability does not imply global external stabilizability for small sensor disturbances, IEEE Trans Automat Control 40:2119-2122

7. Freeman R (1997) Time-varying feedback for the global stabilization of nonlinear systems with measurement errors. In: Proceedings of the European Conference on Control Brussels

8. Freeman R, Kokotovic P (1993) Global robustness of nonlinear systems to state measurement disturbances. In: Proceedings of the 32nd IEEE Conference on Decision and Control San Antonio TX

9. Khalil H (1996) Nonlinear systems Second Edition. Prentice-Hall Upper Saddle River NJ

10. Kokotović P, Arcak M (1999) Constructive nonlinear control progress in the 90's. In: Proceedings of the 14th IFAC World Congress, Plenary and Index Volume Beijing

11. Krstic M, Deng H (1998) Stabilization of uncertain nonlinear systems. Springer London

12. Krstic M, Kanellakopoulos I, Kokotović P (1995) Nonlinear and adaptive control design. John Wiley and Sons New York

13. Liberzon D, Sontag E, Wang Y (2002) Universal construction of feedback laws achieving ISS and integral-ISS disturbance attenuation, Systems Control Lett 46:111-127

14. Malisoff M, Rifford L, Sontag E (2003) Global asymptotic controllability implies input-to-state stabilization, SIAM J Control Optim to appear

15. Malisoff M, Sontag E (2000) Universal formulas for feedback stabilization with respect to Minkowski balls, Systems Control Lett 40:247-260 
16. Rifford L (2000) Problèmes de stabilisation en théorie du contrôle, Thèse Université Claude Bernard Lyon I

17. Rifford L (2000) Existence of Lipschitz and semiconcave control-Lyapunov functions, SIAM J Control Optim 39:1043-1064

18. Rifford L (2002) Semiconcave control-Lyapunov functions and stabilizing feedbacks, SIAM J Control Optim 41:659-681

19. Sontag E (1983) A Lyapunov-like characterization of asymptotic controllability, SIAM J Control Optim 21:462-471

20. Sontag E (1989) Smooth stabilization implies coprime factorization, IEEE Trans Automat Control 34:435-443

21. Sontag E (1989) A 'universal' construction of Artstein's theorem on nonlinear stabilization, Systems Control Lett 13:117-123

22. Sontag E (1990) Further facts about input to state stabilization, IEEE Trans Automat Control 35:473-476

23. Sontag E (1998) Comments on integral variants of ISS, Systems Control Lett 34:93-100

24. Sontag E (1998) Mathematical Control Theory Second Edition. Springer New York

25. Sontag E (1999) Stability and stabilization discontinuities and the effect of disturbances. In: Clarke F, Stern R (eds) Nonlinear Analysis Differential Equations and Control. Kluwer Dordrecht

26. Sontag E (2000) The ISS philosophy as a unifying framework for stability-like behavior. In: Isidori A, Lamnabhi-Lagarrigue F, Respondek W (eds) Nonlinear Control in the Year 2000 Volume 2. Springer-Verlag Berlin 